\title{
Computational simulation of gliomas using stochastic methods
}

\author{
Otávio Xavier Barbosa ${ }^{1}$ (D), Weslley Luiz da Silva Assis $^{2}$ (D), Vanessa da Silva \\ Garcia $^{3}$ \& Gustavo Benitez Alvarez ${ }^{2}$
}

(1) Universidade Federal Fluminense, Programa de Pós-Graduação em Modelagem Computacional em Ciência e Tecnologia, Avenida dos Trabalhadores 420, Vila Santa Cecília 27225-125, Volta Redonda, Rio de Janeiro, Brazil. E-mail: otavioxb@gmail.com

(2) Universidade Federal Fluminense, Departamento de Ciências Exatas, Programa de Pós-Graduação em Modelagem Computacional em Ciência e Tecnologia, Avenida dos Trabalhadores 420, Vila Santa Cecília 27225-125, Volta Redonda, Rio de Janeiro, Brazil. E-mail: weslleyassis@id.uff.br, benitez.gustavo@gmail.com

(3) Universidade Federal Fluminense, Departamento de Administração, Programa de Pós-Graduação em Modelagem Computacional em Ciência e Tecnologia, Rua Desembargador Ellis Hermydio Figueira, Aterrado 27213-145, Volta Redonda, Rio de Janeiro, Brazil. E-mail: vanessa.sgarcia@gmail.com

Barbosa O.X., Assis W.L.S., Garcia V.S. \& Alvarez G.B. (2019) Computational simulation of gliomas using stochastic methods. Pesquisa e Ensino em Ciências Exatas e da Natureza, 3(2): 199-215.

http://dx.doi.org/10.29215/pecen.v3i2.1285

Academic Editor: Saulo Pomponet Oliveira. Received: 21 September 2018. Accepted: 18 November 2019. Published: 10 December 2019.

\section{Simulação computacional de gliomas via métodos estocásticos}

Resumo: Gliomas são tumores cerebrais primários agressivos e invasivos, no qual o mais comum e maligno, glioblastoma multiforme, possui uma combinação de rápido crescimento e invasibilidade. Com o avanço na capacidade de processamento e armazenamento de dados, a utilização de métodos estocásticos para a simulação de problemas físicos reais vem se tornando cada vez mais frequentes. $\mathrm{O}$ objetivo do trabalho é simular computacionalmente o crescimento do glioma resolvendo uma equação de reaçãodifusão em 1D, pelo método de Crank-Nicolson e transpor essa solução para uma geometria 3D por meio do método do Cone Causal e de Monte Carlo. Os resultados obtidos fornecem informações da evolução do raio, concentração de células cancerosas, volume e uma visualização em 3D do tumor. Estes resultados encontrados se mostraram satisfatórios quando comparado com trabalhos que estudam o crescimento tumoral.

Palavras chave: Equação de Reação-Difusão, Gliomas, Método do Cone Causal, Método de Monte Carlo, Método de Diferenças Finitas.

Abstract: Gliomas are aggressive and invasive primary brain tumors in which the most common and malignant glioblastoma multiforme has a combination of rapid growth and invasiveness. With the advancement in data processing and storage capacity, the use of stochastic methods to simulate real physical problems is becoming more and more frequent. The objective of this work is to simulate computationally the growth of the glioma solving a 1D reaction-diffusion equation, by Crank-Nicolson method, and to transpose that solution to a 3D geometry employing the Causal-Cone and Monte-Carlo methods. The results provide information on the evolution of the radius, concentration of cancer cells, volume and a $3 \mathrm{D}$ visualization of the tumor. These results were found to be satisfactory when compared to studies of tumor growth.

Key words: Reaction-diffusion Equation, Gliomas, Causal Cone Method, Monte Carlo Method, Finite Differences Method. 


\section{Introduction}

Gliomas are malignant tumors that usually occur in the upper cerebral hemisphere but can be found throughout the brain. They originate in the glial cells which are cells of the adjacent brain tissue. Gliomas are characterized by proliferating and invading normal-looking tissue (Murray 2003). They initially grow without manifesting symptoms, and the symptoms depend on the size and location of the tumor. This makes it difficult treating, because they are usually detected when their invasiveness and aggressiveness are too high. Conventional treatment in Brazil includes surgical resection, radiotherapy, and chemotherapy. Even with these treatments, multiform glioblastomas have a tendency to recur and to be fatal with an average survival of approximately 1 year (Deisboeck \& Stamatakos 2010). The current technological advancement in radiological medical images offers non-invasive imaging techniques that allow the detection of the brain's anatomy tumors as anomalies in molecular activities (Deisboeck \& Stamatakos 2010). An example of such exams is magnetic resonance imaging and positron emission tomography.

Mathematical models have recently been proposed to help understand the spatial and temporal distribution of gliomas. These models can be divided into three approaches: continuous, discrete and hybrid models. According to Swanson et al. (2003), mathematical modeling is a powerful tool to analyze biological problems, allowing to develop and test hypotheses that can lead to a better understanding of the biological process. With advances in data storage and processing capacity, computational modeling has gained considerable strength in the study of gliomas. Computational models describing the dynamics of gliomas were initiated in the 1990s by Smolle (1993), Tracqui et al. (1995) and Cruywagen et al. (1995). These first articles were aimed at modeling the effect of chemotherapy and surgical resection on the evolution of glioblastomas multiforme. This paper focuses on the study of the continuous macroscopic model proposed in Swanson et al. (2003) which describes the growth and spread of tumor cell density at a macroscopic level. The reactive-diffusive partial differential equation (PDE) takes into account the evolution of the tumor cell population as continuous variables, excluding the discrete character of cells (Junior 2003). The diffusive term of the PDE models the invasiveness of the glioma, while the reactive term describes the proliferation of cells.

The major contribution of this work is to propose a new methodology for simulating the growth of glioblastomas multiforme in three dimensions by solving a reactive-diffusive PDE in one dimension. This methodology is composed of three fundamental elements: the reactivediffusive PDE in one dimension, the Causal Cone method, and the Monte Carlo method. The reactive-diffusive equation proposed by Swanson et al. (2003) was used to describe the dynamics of tumor cells. It was solved by the finite difference method for the discretization of the spatial variable and the Crank-Nicolson method for the discretization of the temporal variable. In this way, we obtained the concentrations of cancer cells for each instant of time in all points of the one-dimensional mesh. It is known that reactive-diffusive kind models assume the existence of spherical symmetry (Murray 2003), although this is an approximation that allows simplifying the mathematical model. This hypothesis of spherical symmetry allows the use of the Causal Cone method (Cahn 1996). Thus, it is possible to generate the spherical volume of the tumor from the glioma radius without having to solve a PDE in three dimensions. On the other hand, the technology that generates medical images available at the present establishes a visual resolution of approximately $1 \mathrm{~mm}$. This suggests that there is some degree of randomness in defining the border of the tumor. Because of this inaccuracy in the actual size of the tumor, when radiotherapy is used, it is common to irradiate a spherical region with a radius between 1 and $3 \mathrm{~cm}$ greater than the visible region (safety margin). Based on this fact the Monte Carlo method will be used to generate a non-uniform volume of the tumor according to the nonuniform distribution of cancerous cells.

\section{Methodology}

\section{Mathematical Model of Glioma}


The model presented by Murray (2003), which describes the dynamics of glioma growth in a homogeneous medium, is represented by a reaction-diffusion equation. The equation states that the rate of time variation of tumor cell concentration is equal to the diffusion migration of tumor cells plus the proliferation of cells.

$$
\left\{\begin{array}{rc}
\frac{\partial c}{\partial t}=\nabla \cdot(D \nabla c)+\rho c & \text { in } \Omega \\
c(\mathbf{x}, 0)=c_{0}(\mathbf{x}) \text { and } \mathbf{n} \cdot \nabla c=0 & \text { on } \partial \Omega
\end{array},\right.
$$

where $c(\mathbf{x}, t)$ is the concentration of cells per $\mathrm{cm}^{3}$ in the point $\boldsymbol{x}$ and at the instant of time $t, D$ represents the mobility of cancer cells measured in $\mathrm{cm}^{2} /$ day, $\rho$ is the cell proliferation rate measured at 1/day, $c_{0}(\mathbf{x})$ is the initial concentration of cancer cells at the point $\boldsymbol{x}$, and $\mathbf{n}$ is the unit vector normal to the boundary $\partial \Omega$. The boundary condition $\mathbf{n} \cdot \nabla c=0$ means that there are no tumor cells migrating out of the skull defined by the region $\Omega \cup \partial \Omega$.

\section{Non-Dimensional Mathematical Model}

The methodology proposed in this work makes use equation (1) in one dimension (1D) to obtain simulations in three dimensions (3D). In this context, the spatial domain is given by $x$ $\in[0, L]$, where $L=20 \mathrm{~cm}$ is adopted as the average length of a human skull. In order to construct a computational code that solves numerically the problem described above it is convenient to write equation (1) in a dimensionless form. Here, we follow the same dimensionless performed in da Silva (2014) and Rockne et al. (2009). Thus, the new dimensionless variables are $\bar{x}=x / L, \bar{t}$ $=\rho t$ and $\bar{c}=\mathrm{c} L^{3} / \hat{c}_{0}$. Then, the computational spatial domain is presented as $\bar{x} \in[0,1]$ and the time domain as $\bar{t} \in\left[0, \rho t_{f}\right]$. The equation (1) can be rewritten in the form

$$
\frac{\partial \bar{c}}{\partial \bar{t}}=D^{*} \nabla^{2} \bar{c}+\bar{c}
$$

where $D^{*}=D / \rho L^{2}$. The initial condition imposed on the problem, following da Silva (2014) and Rockne et al. (2009) ideas, states that there is an initial concentration of cells defined by

$$
\bar{c}(\bar{x}, 0)=\left(L^{6} / \widehat{c}_{0}\right) e^{-\left(100 / L^{2}\right) \bar{x}^{2}} .
$$

The corresponding boundary condition, $\mathbf{n} \cdot \nabla \bar{c}=0$, models the fact that glioma rarely metastasizes. That is, there is no tumor migrating out of the brain.

\section{Finite Difference Method}

The equation (2) in 1D was solved using Crank-Nicolson method that discretizes the temporal part of the model through the forward finite difference formula of the first order, nevertheless the spatial part was approximated by the average of each finite difference formula centered of second order in the time step " $k+1$ ” and in the previous time step " $k$ ", likewise as it was used in da Silva (2014), de Jesus (2014) and de Souza (2015).

The spatial domain is discretized in $M$ intervals with $\Delta \bar{x}=\left(\bar{x}_{f}-\bar{x}_{1}\right) / M$, and the time domain was divided into $K$ intervals with $\Delta \bar{t}=\left(\bar{t}_{f}-\bar{t}_{1}\right) / K$, where " 1 " represents the starting point and " $f$ " the endpoint of each domain. Thus, the equation (2) is approximated by

$$
\bar{C}_{m}^{k+1}-\bar{C}_{m}^{k}=\frac{\lambda}{2}\left(\bar{C}_{m+1}^{k+1}-2 \bar{C}_{m}^{k+1}+\bar{C}_{m-1}^{k+1}+\bar{C}_{m+1}^{k}-2 \bar{C}_{m}^{k}+\bar{C}_{m-1}^{k}\right)+\frac{\Delta \bar{t}}{2}\left(\bar{C}_{m}^{k+1}+\bar{C}_{m}^{k}\right)
$$


where $\lambda=\frac{D^{*} \Delta \bar{t}}{\Delta \bar{x}^{2}}$. The approximate equation for the boundary condition takes the form

- First point of the space mesh $(m=1)$

$$
\frac{1}{2}\left(\frac{-3 \bar{C}_{1}^{k+1}+4 \bar{C}_{2}^{k+1}-\bar{C}_{3}^{k+1}}{2 \Delta \bar{x}}+\frac{-3 \bar{C}_{1}^{k}+4 \bar{C}_{2}^{k}-\bar{C}_{3}^{k}}{2 \Delta \bar{x}}\right)=0
$$

- Last point of the space mesh $(m=M+1)$

$$
\frac{1}{2}\left(\frac{3 \bar{C}_{M+1}^{k+1}-4 \bar{C}_{M}^{k+1}+\bar{C}_{M-1}^{k+1}}{2 \Delta \bar{x}}+\frac{3 \bar{C}_{M+1}^{k}-4 \bar{C}_{M}^{k}+\bar{C}_{M-1}^{k}}{2 \Delta \bar{x}}\right)=0
$$

With the discretization (4), (5) and (6) for each spatial node $m$ a system of linear equations is formed at each step of time (da Silva 2014; de Jesus 2014; de Souza 2015), which can be represented as follows

$$
\begin{gathered}
\mathbf{A X}{ }^{k+1}=\mathbf{b}^{k} \\
\mathbf{b}^{k}=\mathbf{E X}^{k}
\end{gathered}
$$

where the matrices $\mathbf{A}$ and $\mathbf{E}$ are tridiagonal. It may be noted that in each iteration in time the matrix $\mathbf{E}$ is first multiplied with the solution vector in the previous time step $\mathbf{X}^{k}$. After that, the linear system is solved.

\section{Stability Analysis of the Numerical Method}

When we deal with problems that vary with time, it becomes indispensable controlling the errors resulting from the approximations made in the model. Following this idea, it is possible to have an estimate of how the approximate solution of the numerical method behaves over time (Hirsch 2007). Here the Von Neumann stability analysis method was used to analyze error growth. The choice of this method of analysis was due to the fact that it is possible to remove the boundary conditions for the stability analysis of linear problems (Franco 2007; da Silva 2014). The method establishes that the numerical solution $S_{n}$ is given by the exact solution $S_{e}$ of the problem plus an error $\varepsilon$ from approximations

$$
S_{n}=S_{e}+\varepsilon
$$

The Von Neumann method considers that the error can be expressed in terms of an expansion in Fourier series

$$
\varepsilon(\bar{x}, \bar{t})=\sum_{m=1}^{M} e^{a \bar{t}} e^{i \kappa \bar{x}}=\sum_{m=1}^{M} \widehat{\varepsilon}(\bar{x}, \bar{t})
$$

where $a$ is a constant, $\kappa=\pi m / L$ is the wave number, $M=L / \Delta \bar{x}=\left(\bar{x}_{f}-\bar{x}_{1}\right) / \Delta \bar{x}$ and $i=\sqrt{-1}$. 
However, the equation for the error is linear, so we can substitute any of the terms in the equation. This means that each component of the series exhibits the same behavior as the series as a whole. Therefore, it suffices to study the term

$$
\hat{\varepsilon}(\bar{x}, \bar{t})=e^{a \bar{t}} e^{i k \bar{x}}
$$

To determine the conditions under which the growth factor of the error $(G)$ remains controlled, we replace the error (11) in the discrete equation that describes the phenomenon under study (4). In order for the solution to be stable over the iterations over time it is necessary that the error decrease with each new iteration, $\mid G \leq 1$. Thus, for the Crank-Nicolson method we arrive at

$$
G=e^{a \Delta \bar{t}}=\frac{1-\left[\lambda(1-\cos \theta)-\left(\frac{\Delta \bar{t}}{2}\right)\right]}{1+\left[\lambda(1-\cos \theta)-\left(\frac{\Delta \bar{t}}{2}\right)\right]}
$$

where $\lambda=\frac{D^{*} \Delta \bar{t}}{\Delta \bar{x}^{2}}, \theta=\kappa \Delta \bar{x}$. In order to satisfy the stability condition it is necessary to have

$$
\lambda(1-\cos \theta)-\left(\frac{\Delta \bar{t}}{2}\right) \geq 0
$$

It can be noted that there will be instability mainly for the worst case, when $\cos \theta=1$. A more detailed stability analysis for this problem is done in da Silva (2014). Figure 1 shows a graphical study of the equation (12) for three cases: G1 with $\Delta \bar{t}=0.01$ and $\lambda=0.4$, G2 with $\Delta \bar{t}=$ 0.002 and $\lambda=0.09$, G3 with $\Delta \bar{t}=0.5$ and $\lambda=0.09$. It shows that with appropriate choices for $\Delta \bar{t}$ and $\lambda$ it is possible to get $|G|$ close to 1 . In particular, values of the order of $10^{-3}$ for $\Delta \bar{t}$ and $\Delta \bar{x}$ guarantee the stability of the Crank-Nicolson method for the values $D$ and $\rho$ considered in this work.

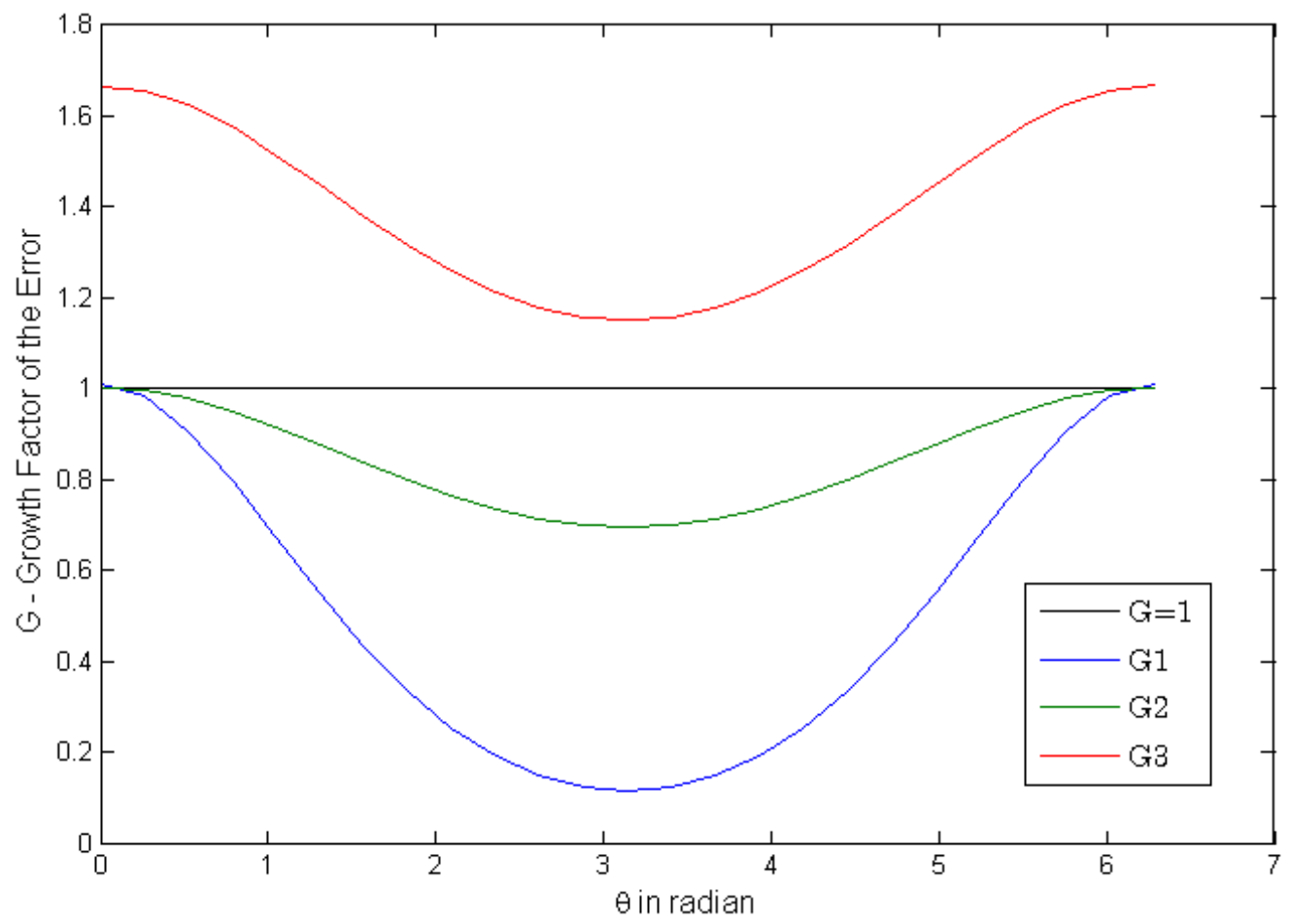

Figure 1. Growth factor of the error as a function of $\boldsymbol{\theta}$. 


\section{Glioma Radius Calculation}

In the macroscopic study of gliomas, its shape is adopted as a spheroid, and this approximation as to its geometry is justified by the results obtained in magnetic resonance imaging. The radius calculation is done through the detectable tumor margin which is 40000 cells $/ \mathrm{cm}^{2}$ or $61.26 \%$ of the initial tumor concentration (de Souza 2015 ).

$$
r(\bar{t})=\left\{\begin{array}{lll}
\bar{x} & \text { if } & \frac{\bar{c}(\bar{x}, \bar{t})}{L^{3}} \geq 0.6126 \\
0 & \text { if } & \frac{\bar{c}(\bar{x}, \bar{t})}{L^{3}}<0.6126
\end{array}\right.
$$

In this mode, the program traverses the positions of the concentration vector at each step of time looking at which position of the spatial domain is the radius of the tumor.

\section{Stochastic Methods}

Stochastic models may represent situations in which there is occasionally an uncertainty factor, that is, they are models for processes that present certain randomness (Nelson 2010). The stochastic word comes from the Greek word stokhazesthai which means pointing or guessing. In nature, uncertainty is part of everyday life, so stochastic models can, in fact, represent a large number of real-life phenomena.

A stochastic model usually can reflect much of the information of the real problem studied, where probabilities are assigned to each specific event of the study, and these probabilities allow us to make future predictions or provide complementary information of the phenomena (Brzezniak \& Zastawniak 2005).

Stochastic processes are ubiquitous in biological systems. In particular, the interface of cancer cells with surrounding healthy tissue provides a relevant scenario for stochastic processes (Rodríguez et al. 2017). In the present work, the stochastic methods of the Causal Cone and Monte Carlo were used.

\section{Causal Cone Method}

One of the first uses of this method was seen in the area of physical metallurgy in Cahn (1996). In this work, the Causal Cone Method (CCM) was used to simulate nucleation reactions and growth in a discrete domain. In Cahn's study, the CCM was used in contrast to the JMAK theory (Kolmogorov-Johnson-Mehl-Avrami), which takes into account factors such as "ghost regions" and "extended volumes". These factors are constructs that allow exact estimates of the fraction transformed into a sample as a function of time. With the use of CCM there is no need to use such factors.

Usually, stochastic models depend on a transition rule to simulate dynamic evolutions. The transition rules are responsible for changing or not the state of each cell of the matrix (discrete domain) at each discrete time (step) during the computational simulation. The Causal Cone theory, in a three-dimensional view, says that there is at least one nucleus (tumor) transformed into a point of the matrix within the sphere of radius $\mathrm{R}$, centered at that point. As the radius increases with each iteration, the volume of the sphere increases and the probability of nuclei within the sphere also increases (Ribeiro 2011). The junction of all spheres that evolve over time creates a "Cone in the Time domain," and for this reason, the model is known as the Causal Cone or Time Cone. So there is a problem in four dimensions, three spatial dimensions and a temporal dimension. It is relevant to say that each isolated point within the matrix has its independent "cone" (Ribeiro 2011).

Figure 2 is a direct illustration for this situation mentioned in the previous paragraph. However, it is important to emphasize that to facilitate the visualization and better 
comprehension, two spatial dimensions, and a temporal dimension were used. They are the concentration of cancer cells and the detectable radius of the tumor.

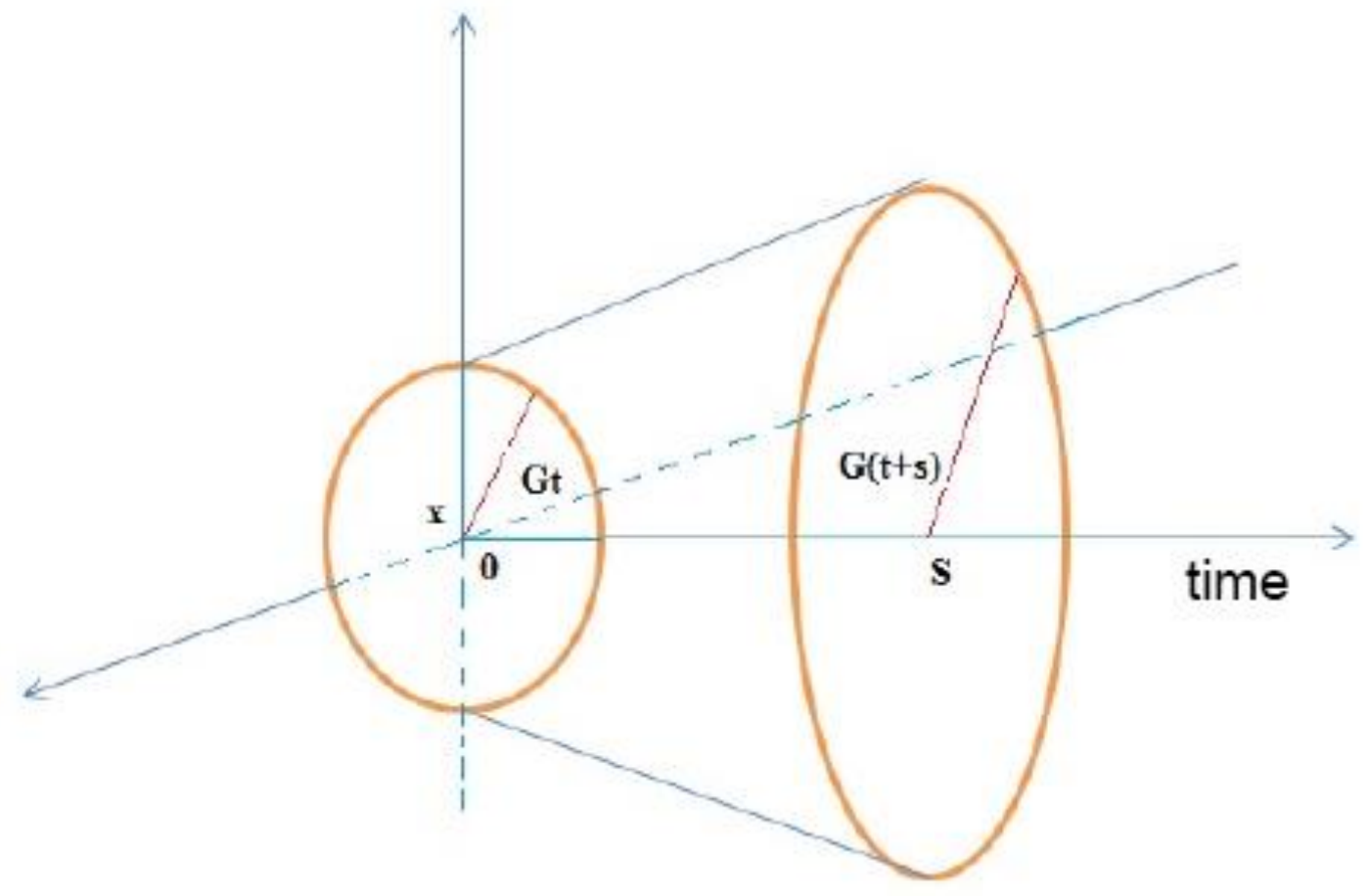

Figure 2. Three-dimensional representation of the Causal Cone. Source: Ribeiro (2011).

As already mentioned in this work the representation of each tumor volume can be described geometrically by a massive sphere. The Causal Cone method is fundamental in this work because it is in charge of making this geometric representation in space, saving the coordinates and states (values) of the concentrations as a function of time during the evolution of the glioma, and also returns the value of the radius to margin detectable according to the concentration at that time. Moreover, the Causal Cone method acts as a limiting boundary for the Monte Carlo method, in the sense that there can be no concentration values outside the sphere generated at any given time point $t$ under analysis. In general, any validated method or algorithm that represents the discrete case of a growing sphere could be used in this work.

Causal Cone Method was chosen due to some favorable and very particular factors for this study. First, the CCM is well suited for mobile boundary problems, where the boundaries of two or more distinct regions lie within the discrete domain. Most numerical spherical growth models have some restrictions when dealing with the impingement phenomenon or clashes between two adjacent interfaces. Another advantage is that CCM is a highly parallelizable method. Parallelization is a feature that is very useful in computational simulation. Another specific advantage for this work was to have a discrete model already validated by CCM prepared to simulate nucleation and growth. The base of the computational code whose Causal Cone Method was previously implemented and publicized in other works of international journals (Alves et al. 2018; da Fonseca et al. 2018; Ventura et al. 2018) was also used to develop part of the numerical model proposed in this study. At each iteration a new radius value is calculated, thus, the glioma evolves as a function of the discrete time also known as step. For discrete evolution to take place at each step the CCM model needs to be fed by a new radius increment $\Delta r$ to calculate the new volume of the spheres at each point of the mesh. After this calculation, the analysis is performed if there is at least one nucleus within the sphere generated from the point analyzed in the mesh. If the result of the check is true, then that tested point that generated the sphere of radius " $r$ ", will also transform (change state). In the proposed numerical model, the program uses the values of the tumor radii calculated using equation (14). From these radii, it is 
possible to calculate the volumes of the glioma from each iteration. The Causal Cone Method depends on a new radius to generate a new time cone at each simulation step. The radii calculated using equation (14) in this work are used as input data to feed the CCM. In a classic CCM, a time cone is generated on each mesh node, so it is fair to search for a nucleus that is within that cone at that particular moment.

The problem studied in this work is the growth of tumors, the method does not need to look for where there are nuclei. The cancerous tumor is concentrated in a specific region of the space, so, for each radius value obtained by the detectable margin of the glioma, the computational algorithm will transform all the internal points near the tumor with distances smaller than the radius at that time. Thus, with each increase in radius value, the algorithm transforms all existing points between the previous radius and the current radius, generating a new sphere of greater volume than the previous one. During the evolution of the glioma volume, concentration also evolves as a function of time. At each step the concentration of cancer cells increases according to the values obtained by equation (1). In short, the concentration of each point of the mesh is recalculated and updated. This concentration update is transferred to a new mesh, with dimensions equivalent to the mesh generated by the Causal Cone Method. This new mesh is a mirror of the Time Cone mesh, but in this mesh is saved the values of concentration. In this way, we obtain two meshes that represent the evolutionary process of the glioma. The first mesh shows the geometry and size of the glioma within the discrete domain, the second mesh saves concentration values throughout the glioblastoma growth. This process repeats as new radius and concentration values are passed to the Causal Cone.

At the end of this process, the computational code provides concentric spherical shells transposing the initial domain from one dimension to a three-dimensional domain. Figure 3 illustrates the growth of the glioma for different values of radius, obtained through the Causal Cone method. According to Murray (2003) the shape of the gliomas can be adopted as spheroids, so the Causal Cone method can be considered a good choice. This choice is due not only to the fact that the method simulates the growth of spheres during an evolutionary process, although also to the advantages mentioned above, such as the flexibility of the method, presenting good results in problems evolving interaction between two moving interfaces, the implementation can be validated by exact analytical solution, and to be highly parallelizable. The application of the solution of geometric representation and concentration via Time Cone allows the implementation of the growth of two or more tumors simultaneously growing in different regions. For example, in the case of glioma two regions adjacent to each other: a region of gray matter and another region of white matter. In materials science, metallurgy and stochastic mathematics, some situations analogous to this have already been studied via CCM in the works da Alves et al. (2018), da Fonseca et al. (2018) and Ventura et al. (2018).

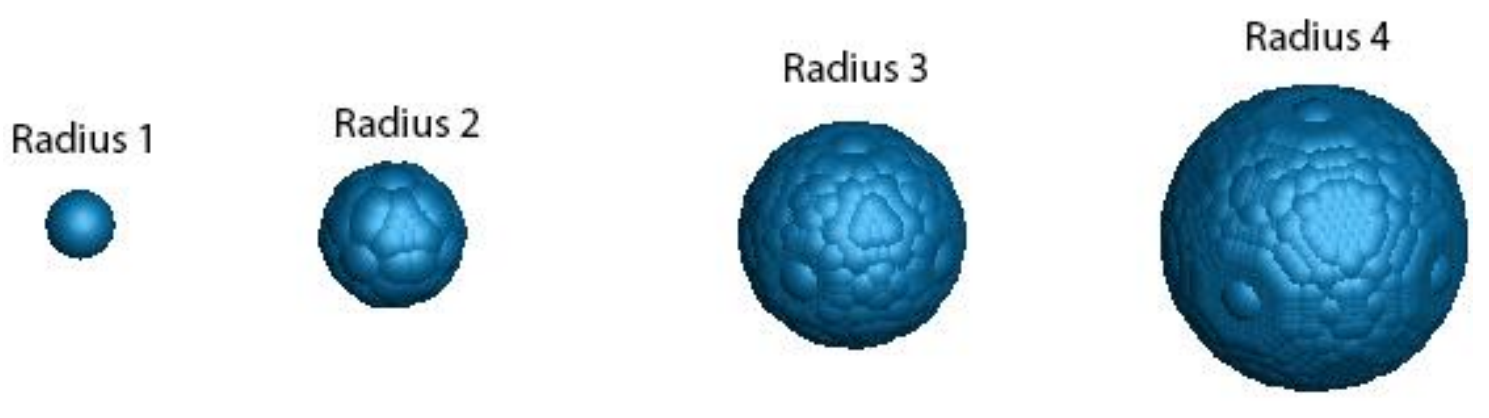

Figure 3. Transposition of the 1D domain to 3D by the Causal Cone method.

The volume of the Causal Cone, presented by Alves (2015) can be calculated by the time-adjusted polynomial to $t \geq 1$ as 


$$
V(t)=\frac{4 \pi t^{3}}{3}-\frac{1 t^{2}}{22}-\frac{79 t}{30}+\frac{347}{36}
$$

This equation is not valid for $t=0$. It is possible to omit the terms with small influence on the result. This is due to the fact that for very long times (tending to infinity) the terms with degree smaller than two become insignificant when compared with the term of degree three. Thereby, the expression reduces to equation (16)

$$
V(t) \cong \frac{4 \pi t^{3}}{3}
$$

Therefore thus, it can be said that the error of the Causal Cone method is derived from these usually neglected terms in equation (15).

\section{Monte Carlo Method (MCM)}

The Monte Carlo method was formalized in 1949, by article published by John Von Neumann and Stanislav Ulam. The name was given to the random character of the common roulette games in casinos, especially the Monte Carlo casino. Normally, Monte Carlo models can be divided into three characteristic steps. In the first step, the physical problem under investigation is translated into an analogous probabilistic or statistical model. In the second step, the probabilistic model is evaluated by a numerical stochastic sampling experiment, including a large number of arithmetic and logic operations. In the third step, the obtained results are analyzed using statistical methods (LeSar 2013). The method is characterized by the impossibility of having the analytical solution of the problem, but the result is a good approximation of the solution after a large number of samples are processed.

Among the several versions of the existing Monte Carlo method, we have used a model called by some authors as the Kinetic Monte Carlo method. For the application of this, three basic information is needed:

1. Probability Density Function,

2. Random Number Generator,

3. Sampling Techniques.

The probability density function (PDF) represented by $f(x)$ is a measure of observing $x$, so that

- $f(x) \geq 0$, provided there are no negative values,

- $f(x)$ is normalized in the domain of $x$, that is: $\int_{x_{\min }}^{x_{\max }} f(x) d x=1$.

In addition, we have the cumulative density function $F(x)$ defined as

$$
\int_{x_{\min }}^{x} f(x) d x=F(x)
$$


which has the following two properties: $F\left(x_{\min }\right)=0$ at the beginning of the domain, and $F\left(x_{\max }\right)$ $=1$ at the end of the domain.

Every Monte Carlo simulation needs a random number generator. These numbers have randomness in order to approach the occurrence in nature. These numbers belong to an interval, for example [0,1], and are useful in making decisions for physical problems that present a large number of possible outcomes (Yoriyaz 2009).

Given the probability density function that represent the studied physical phenomenon and a random number generator it is possible to establish sampling techniques that relate the PDF and the random numbers $r$. To do this, it is necessary to have a valid PDF $f(x)$ in any interval [a,b]. If this function is integrable in the same interval, then it is possible to obtain the cumulative probability function given by equation (17). If $f(x)$ is normalized we have to $F(b)=$ 1. Since $F(x)$ is in the interval [0,1], it is possible to analyze this cumulative probability function by means of the variable $r$ that is evenly distributed between 0 and 1 , such that $r=F(x)$ or $x=\frac{1}{r}$. Thus, one can find $x$ of the probability density function by means of a random number $r$.

In the present work, the probability density function used was generated from the results obtained by the glioma cell concentration values. The concentration values were obtained by solving equation (1) at each time step. The concentrations have a maximum value of cells in the center that decreases as the distance from the center increases. Thus, by dividing the concentration values at each point of the mesh by the higher concentration value (near the center) a concentration gradient is obtained, which varies from one (in the center) to close to zero the further away. It is shown through Figure 4 an illustration for the probability density function as a function of the tumor radius at any given time.

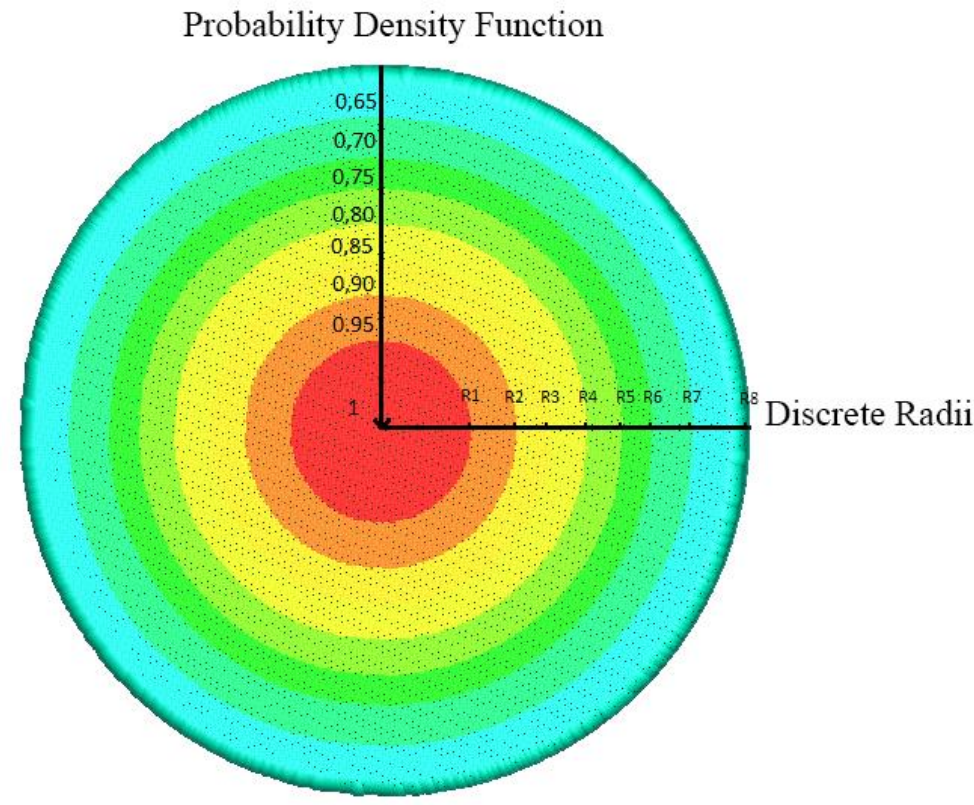

Figure 4. The probability density function as a function of the glioma radius.

Initially, the Causal Cone method implemented in the computational code allows calculating the maximum sphere at which the glioma can reach. Thus, we have the maximum domain that the Monte Carlo method can use to start performing the nucleations. After the PDF is calculated, the computational code randomly draws a point within the sphere obtained by the Causal Cone Method and generates a random number between [0,1]. Subsequently, the following condition is verified: if the random number is less than or equal to the PDF relative to that point, then that point is transformed. Conversely, the algorithm does not transform this point and leaves to another point of the three-dimensional mesh. The above condition is verified for all drawn points within the sphere generated by the CCM in time $t_{0}$. For each different point 
drawn within the sphere, a new random number is generated to test the condition. By increasing time, a new concentration of cells is calculated by providing a new probability density function. Thus, the Monte Carlo method is again applied to the problem by performing the tests for all points of the new spatial domain generated by the Causal Cone method. This process is repeated until it covers the entire temporal domain. The computational domain used contains $300^{3}$ nodes in the computational matrix. Thus, 27 million points total in space. During simulations, at each discrete time, the Monte Carlo random draw process occurs around $\frac{300^{3}}{3}$ times, or approximately $\frac{1}{3}$ of the number of domain nodes to reach the convergence. We can see in Figure 5 a synthetic flowchart of the computational code.

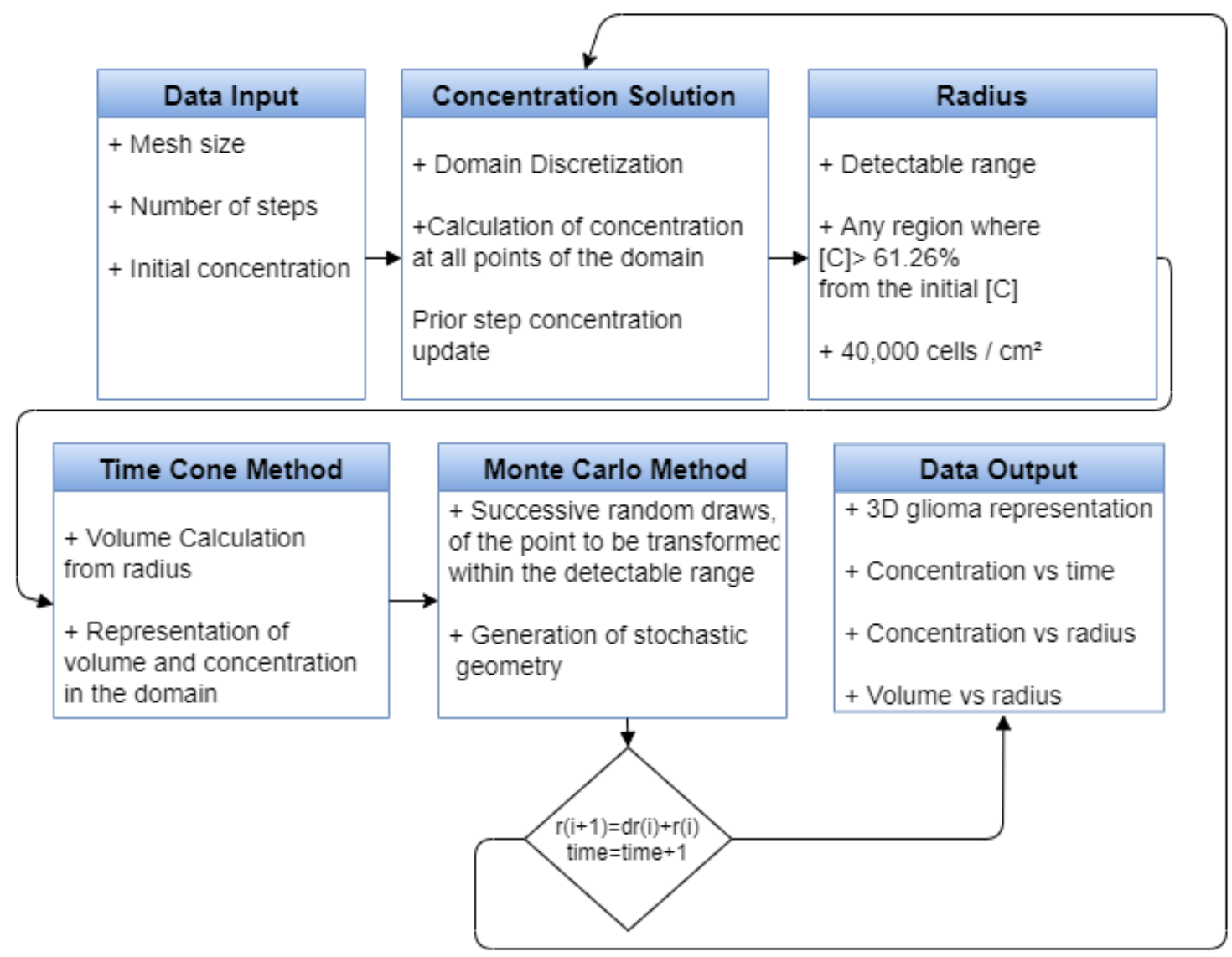

Figure 5. Flowchart of the computational code.

\section{Results and Discussion}

The results shown in this work were calculated on a Samsung notebook, Windows 10, Intel (R) Core i7-3rd Generation, 1 terabyte hard disc, 6 GB of RAM memory, 64-bit operating system and x64 based processor. The average time spent in each simulation was around 15 minutes. The values used in the work for the diffusion and reaction coefficients were $D=0.0065$ $\mathrm{cm}^{2} /$ day and $\rho=0.0121 /$ day (Swanson et al. 2000). Table 1 shows the evolution of the tumor radius over time according to its respective diffusion and proliferation coefficient.

According to Murray (2003), Swanson et al. (2000) and Elaff (2018) the image-detectable radius and the lethal radius are respectively $1.5 \mathrm{~cm}$ e $3.0 \mathrm{~cm}$. The average time to reach the maximum radius, sufficient to kill an individual, is about 80 to 110 days, which is in accordance with the results obtained in the simulations of the present work. Figure $6 \mathrm{~A}$ is an illustration of the data contained in Table 1, and these values obtained by the simulation are in agreement with those found in Swanson et al. (2000). 
Table 1. Temporal evolution of the tumor radius.

\begin{tabular}{cc}
\hline Time (days) & Radius $(\mathbf{c m})$ \\
\hline 0 & 1.40 \\
8 & 1.52 \\
17 & 1.68 \\
25 & 1.82 \\
33 & 1.96 \\
46 & 2.10 \\
50 & 2.24 \\
58 & 2.38 \\
66 & 2.52 \\
75 & 2.64 \\
83 & 2.78 \\
\hline
\end{tabular}

Bellomo et al. (2008) claimed that the volume of the glioma can be approximated to the volume equation of the sphere $V=4 \pi r^{3} / 3$. This approximation is due to the fact that the tumor is considered like a spheroid. Tumor volumes simulated in this work are shown in Figure 6B. The initial volume shows the approximate value of $11.5 \mathrm{~cm}^{3}$ and $92 \mathrm{~cm}^{3}$ for the final volume after 80 days. The values found in this work are in agreement with the mean values of volume found in the literature for the initial detectable volume and for the lethal volume. As discussed by Burgess et al. (1997) the detectable volume is comprised between $10 \mathrm{~cm}^{3}$ to $29 \mathrm{~cm}^{3}$, while the lethal volume of the tumor is within an accepted range of $58 \mathrm{~cm}^{3}$ to $100 \mathrm{~cm}^{3}$.

A
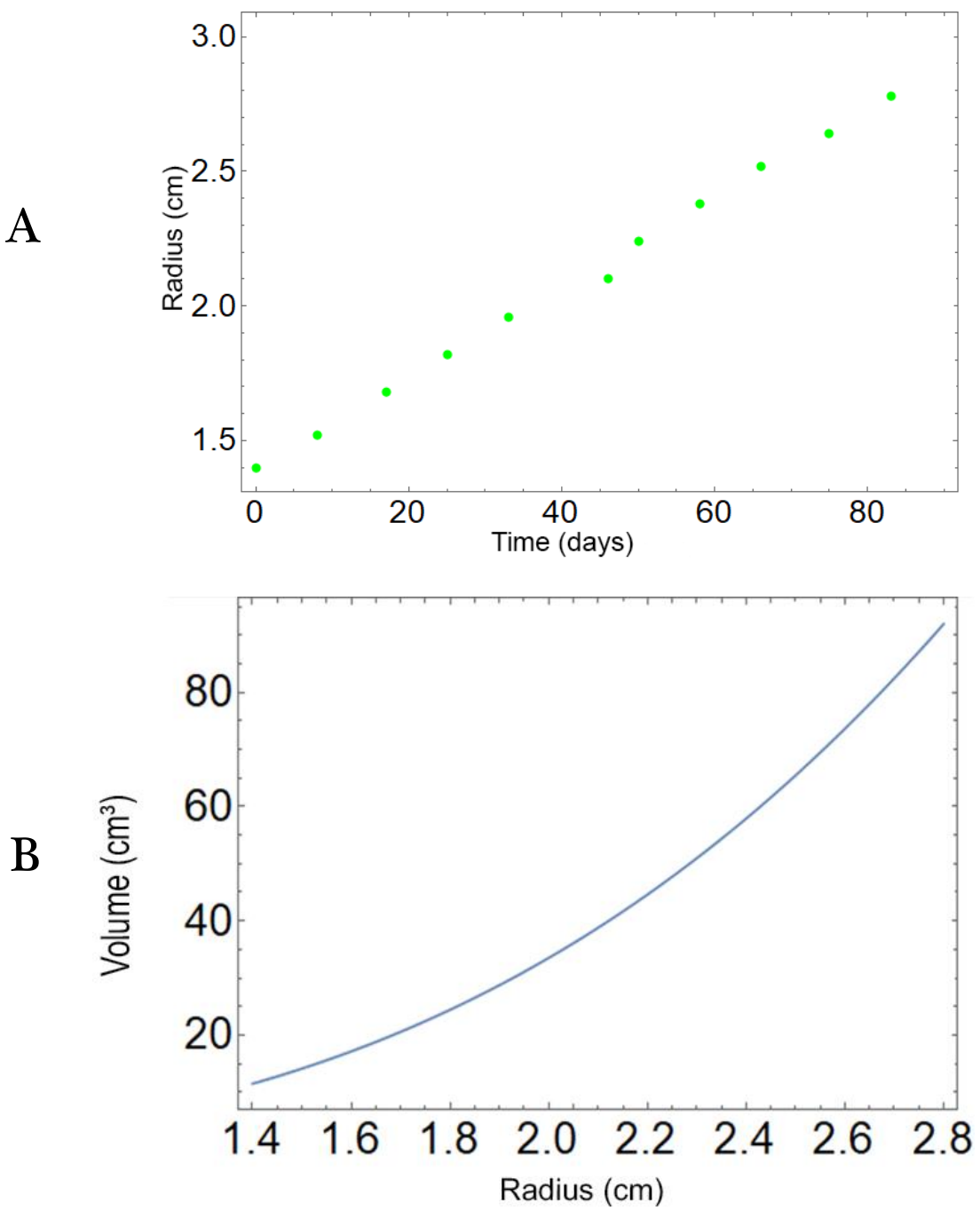

Figure 6. Radius and volume of the tumor as a function of time: A. Radius of the tumor; B. Tumor volume. 
A striking feature of glioblastomas is that instead of forming a solid mass of tumors with well-defined borders, it tends to form a smooth gradient of cell concentrations. Tumor cells are known to infiltrate adjacent brain tissues, which can be found several centimeters apart from the increase in tumor mass visible on MRI images. Although the progress in imaging studies is quite remarkable, it is still not possible to identify areas of low tumor cell infiltration (Unkelbach et al. 2014).

In actual practice, planning for radiotherapy is typically based on gross tumor volume visible on MRI images. Many radiologists consider infiltrative growth by expanding the gross tumor volume with a margin of 1 to $3 \mathrm{~cm}$ to form the target clinical volume, which is irradiated by a homogenous dose of 60 Gy (gray). The current treatment planning procedure can be improved by accounting for two growth characteristics of gliomas: anisotropic tumor growth and tumor cell density that varies spatially (Unkelbach et al. 2014).

For an analysis of the anisotropic behavior of the concentrations in the results presented in this work, it is necessary visualization of the interior of the tumor, because in this manner, it is possible to analyze how the concentrations of cells are being distributed from the center to the border of the glioma. Thus, a cross-section was made in the virtual glioma for a perception of such a characteristic mentioned above.

Two images of the simulated glioma in this work are presented in Figure 7. Figure 7A depicts the concentration distribution by the Causal Cone method, while Figure 7B shows the result obtained using the Causal Cone method in conjunction with the Monte Carlo method. Both images represent the stage of the glioma with the same radius, concentration and instant of time. The difference in the representation of the cell concentrations in each case is perceptible. In Figure 7A we see a smooth sphere, with cell concentration that decreases throughout the radius, but homogeneously. In turn, Figure 7B shows a heterogeneity in the variation of the concentration of cells. The region with the highest concentration and also the highest homogeneity in the concentration is located in the center, but as it approaches the border, fewer points are transformed, decreasing the concentration of cells and increasing the heterogeneity in concentration. The idea of including the Monte Carlo method in our methodology is precisely to be able to represent the concentration gradient presented in Unkelbach et al. (2014). Thus, greater homogeneity of the tumor near the center and a decrease in homogeneity towards the external border of the tumor is noted.
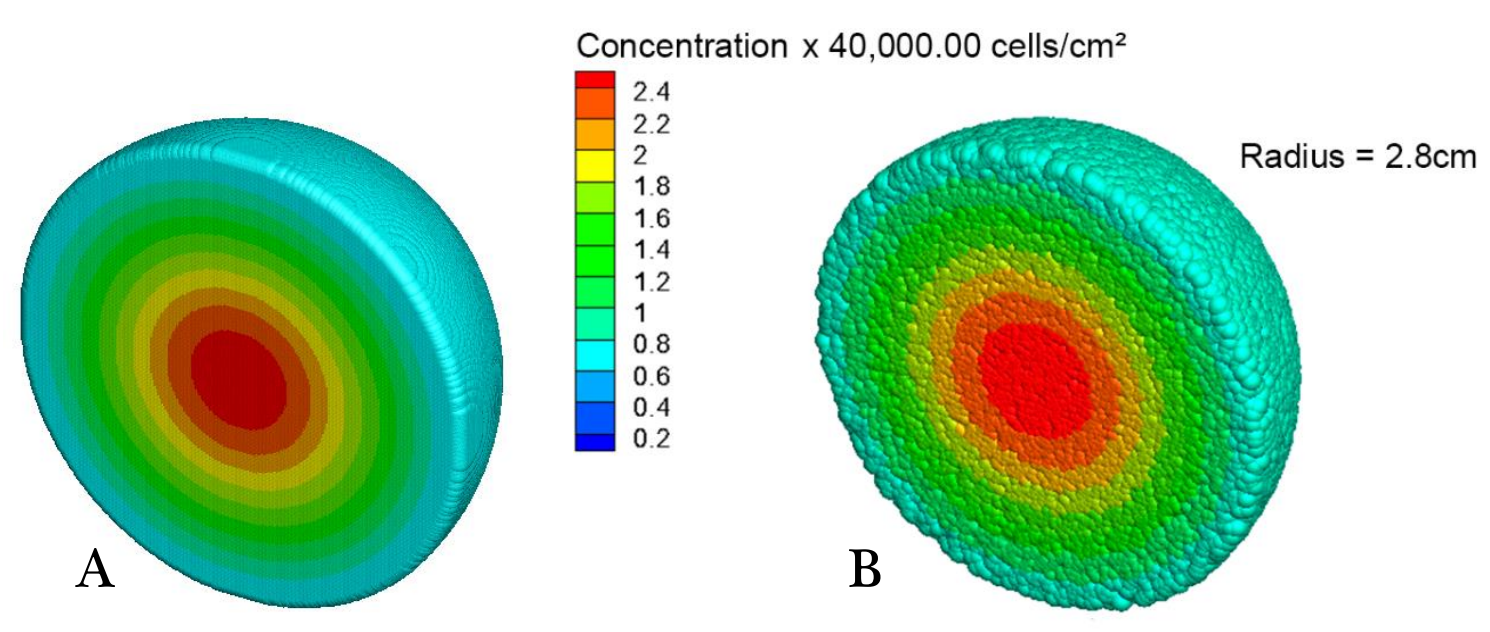

83 days

Figure 7. Homogeneity and heterogeneity in the distribution of cell concentration: A. Causal Cone; B. Causal Cone and Monte Carlo.

The combination of the Monte Carlo and the Causal Cone methods is effective for the representation of gliomas, since according to Bellomo et al. (2008) and Deisboeck \& Stamatakos (2010) the gliomas are considered as spheroids. Thus, the Causal Cone method generates a 
smooth sphere that serves as a delimiter and the Monte Carlo method performs the transformations giving the heterogeneous behavior of the concentrations and generating a sphere with irregular boundaries.

A sequence of glioma images according to the evolution of the radius is shown in Figure 8. A spherical symmetry with irregular boundaries can be noted, as shown in Kansal et al. (2000) and Tanaka et al. (2009). In addition, a concentration gradient is observed, showing a region of greater homogeneity near the center of the tumor that decreases as it moves away from the center of the tumor, similarly as exposed by Chaplain (2008). Usually, magnetic resonance imaging is shown in cross-sections of the brain, providing cell concentration values per unit area, so in the images in Figure 8 concentrations of cells $/ \mathrm{cm}^{2}$ are reported.

Concentration $\times 40,000.00$ cells $/ \mathrm{cm}^{2}$

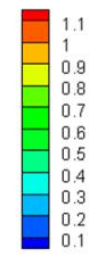

A

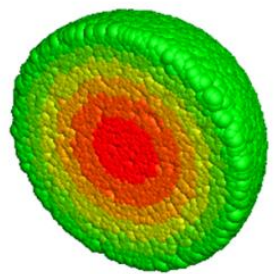

17 days
Radius $=1.7 \mathrm{~cm}$

B
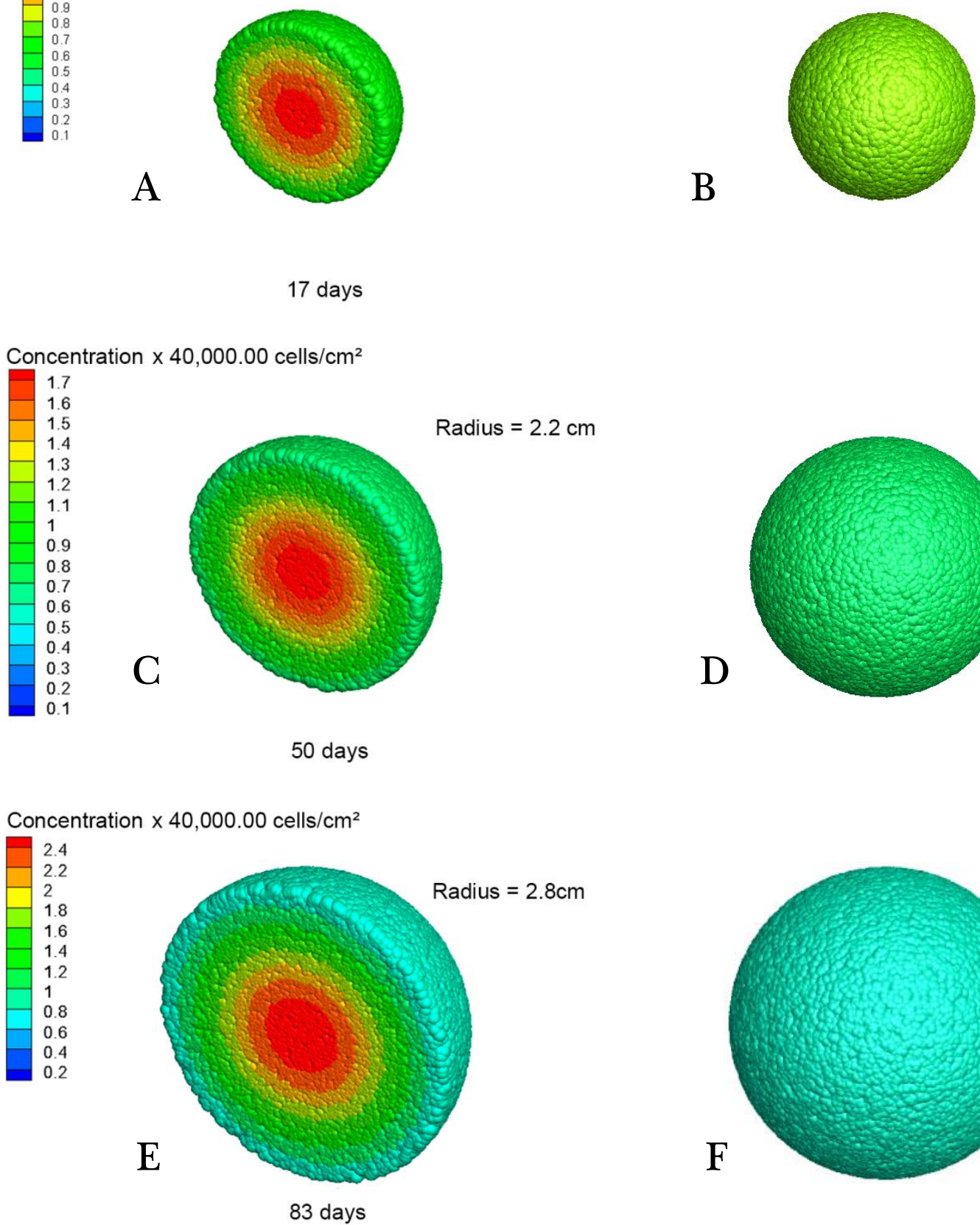

$\mathrm{D}$

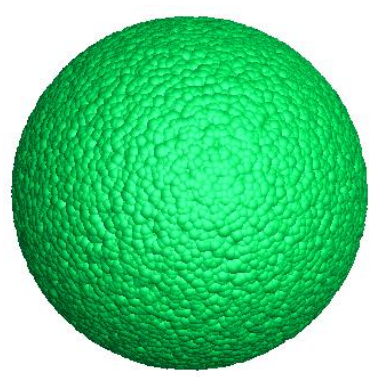

50 days

Radius $=2.2 \mathrm{~cm}$
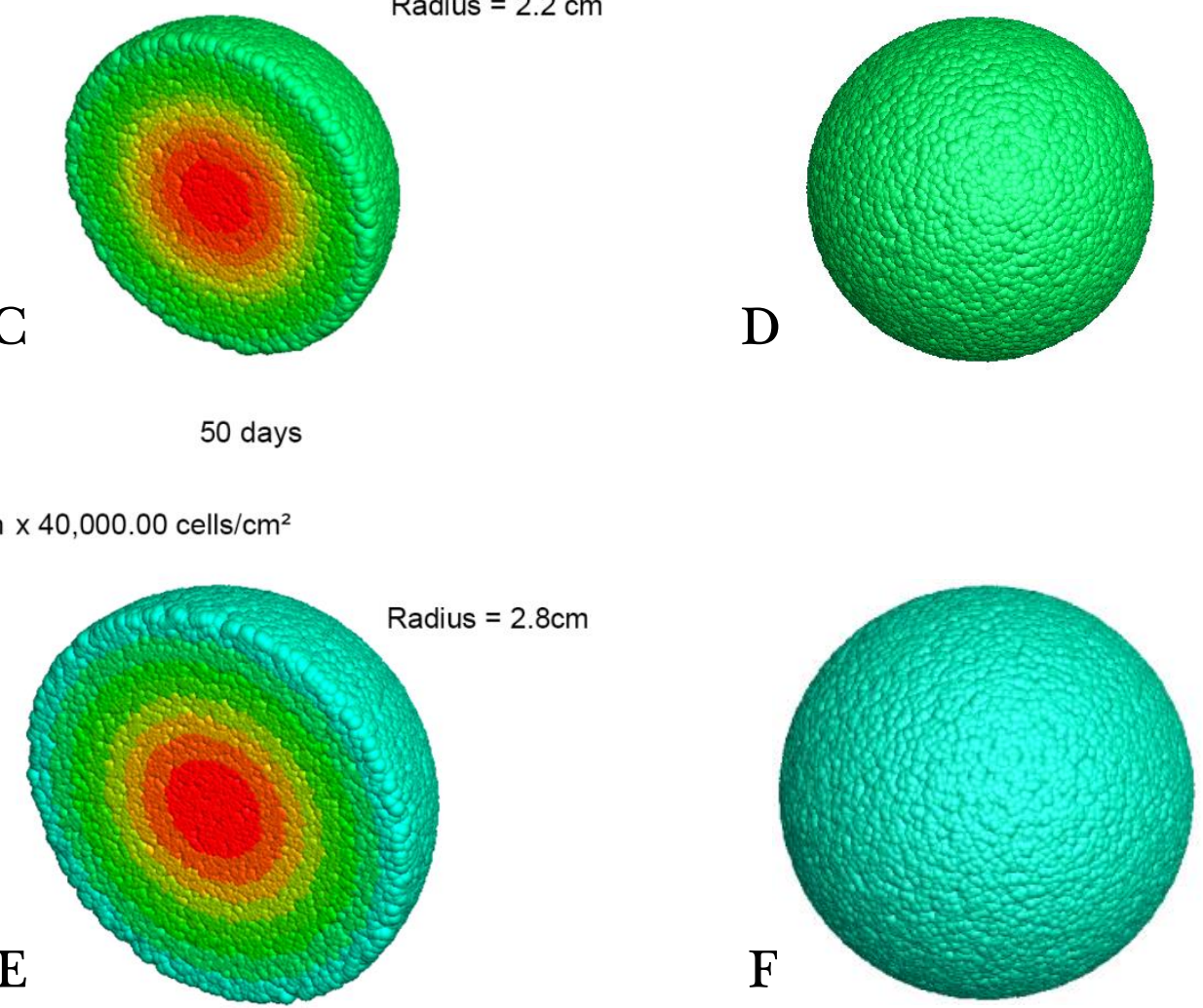

Figure 8. Concentration of cells as a function of the radius: cross section (left) and external view (right): A-B. 49000 cells $/ \mathrm{cm}^{2}$; C-D. 72000 cells $/ \mathrm{cm}^{2}$; E-F. 107000 cells $/ \mathrm{cm}^{2}$. 


\section{Conclusions}

In this work, the growth of gliomas is described by a reaction-diffusion PDE in 1D, which was solved using the Crank-Nicolson method for temporal dependence and the finite difference method for spatial dependence. The numerical solution provided the concentration values for all points of the mesh at different times, which makes it possible to calculate the tumor radius through the detectable margin. The radii calculated at different times are in agreement with the values present in Swanson et al. (2000).

The use of stochastic methods, Cone Causal and Monte Carlo, allowed for the generation of the glioma volume, showing a variation in the heterogeneity of the cell concentration as a function of the tumor radius, thus presenting a gradient of cell concentration showing anisotropic behavior of the concentrations.

The new proposed methodology describes an existing heterogeneity in the growth of gliomas and recognized in the literature. However, the proposed methodology may imply a significantly lower computational cost when compared to models described by PDE in two or three dimensions. This statement can be justified mainly by the following two arguments. First, the matrix system that is given by equation (7) (matrices $\mathbf{A}$ and $\mathbf{E}$ ) is tridiagonal for the $1 \mathrm{D}$ case, whereas in 2D and 3D the same matrices have five and seven diagonals respectively (da Silva 2014). Second, for models described by 2D or 3D PDE to describe the heterogeneity mentioned here, it is necessary to use numerical techniques besides the classic ones presented in this work, which implies increasing computational cost.

Another point to emphasize is that the stochastic methods that form the proposed methodology use only three parameters to perform the nuclei and generate the glioma in three dimensions: the concentration of cancer cells, the tumor radius and a random number generator. However, other stochastic methods studied for the elaboration of the present methodology use at least four distinct parameters (Kansal et al. 2000; Tanaka et al. 2009). This, once again, may indicate a lower computational cost for our methodology.

For future work, it is suggested to include a term related to some therapy, either chemotherapy or radiotherapy (or both together), to follow the evolution of the glioma radius as a function of the treatment. Also, take into account uncertainties in the mobility $D$ and the rate of proliferation $\rho$. With the inclusion of the randomization of these two biological parameters, it is expected that the methodology proposed here will be closer to reality.

\section{Acknowledgment}

The authors thank CAPES financial support. Also, appreciate the comments and suggestions of the reviewers.

\section{References}

Alves A.L.M. (2015) Simulação Computacional de Reações com Nucleação e Crescimento de Fases Simultâneas e Sequenciais pelo Método do Cone Causal. Dissertação de Mestrado, Programa de Pós-Graduação em Engenharia Metalúrgica. Universidade Federal Fluminense, Volta Redonda, Rio de Janeiro.

Alves A.L.M., da Fonseca G.D., da Costa M.F.B., Assis W.L.S. \& Rios P.R. (2018) Evolution of Individual Grains in 3D Micro-structure Generated by Computational Simulation of Transformations Involving Two Phases. Materials Science Forum, 930: 305-310. https://doi.org/10.4028/www.scientific.net/MSF.930.305

Bellomo N., Chaplain M. \& De Angelis E. (2008) Selected Topics in Cancer Modeling: Genesis, Evolution, Immune Competition, and Therapy. Boston: Birkhäuser. 473 p.

Brzezniak Z. \& Zastawniak T. (2005) Basic Stochastic Processes. Springer-Verlag London: Springer. $200 \mathrm{p}$. 
Burgess P.K., Kulesa P.M., Murray J.D. \& Alvord E.C. (1997) The interaction of growth rates and diffusion coefficients in a three-dimensional mathematical model of gliomas. Journal of Neuropathology and Experimental Neurology, 56(6): 704-713. https://doi.org/10.1097/00005072-199756060-00008

Cahn J.W. (1996) The Time Cone Method for Nucleation and Growth Kinetics on a Finite Domain. Materials Research Society Symposium - Proceedings, 398: 425-437. DOI: https://doi.org/10.1557/PROC-398-425

Chaplain M.A.J. (2008) Modelling Aspects of Cancer Growth: Insight from Mathematical and Numerical Analysis and Computational Simulation (p. 147-200). In: Capasso V. \& Lachowicz M. (Eds). Multiscale Problems in the Life Sciences. Lecture Notes in Mathematics. Volume 1940. Heidelberg: Springer. 323 p.

Cruywagen G.C., Tracqui P., Woodward D.E., Cook J., Murray J.D. \& Alvord E.C. (1995) The modeling of diffusive tumours. Journal of Biological Systems, 3: 937-945. https://doi.org/10.1142/S0218339095000836

da Fonseca G.D., Alves A.L.M., da Costa M.F.B., Lyrio M.S., Assis W.L.S. \& Rios P.R. (2018) Modeling and Simulation of Nucleation and Growth Transformations with Nucleation on Interfaces of Kelvin Polihedra Network. Materials Science Forum, 930: 299-304. https://doi.org/10.4028/www.scientific.net/MSF.930.299

Deisboeck T.S. \& Stamatakos G.S. (2010) Multiscale Cancer Modeling. Boca Raton: CRC Press. $484 \mathrm{p}$.

de Jesus J.C. (2014) Modelagem do Crescimento de Glioma por Séries Temporais em Resposta à Radioterapia. Dissertação de Mestrado, Programa de Pós-graduação em Modelagem Computacional em Ciência e Tecnologia. Universidade Federal Fluminense, Volta Redonda, Rio de Janeiro.

da Silva J.J. (2014) Modelagem Computacional Aplicada ao Tratamento de Câncer Via Medicina Nuclear. Dissertação de Mestrado, Programa de Pós-graduação em Modelagem Computacional em Ciência e Tecnologia. Universidade Federal Fluminense, Volta Redonda, Rio de Janeiro.

de Souza É.B. (2015) Otimização do plano de tratamento por radioterapia em pacientes com gliomas via algoritmos genéticos. Dissertação de Mestrado, Programa de Pós-graduação em Modelagem Computacional em Ciência e Tecnologia. Universidade Federal Fluminense, Volta Redonda, Rio de Janeiro.

Elaff I. (2018) Comparative study between spatio-temporal models for brain tumor growth. Biochemical and Biophysical Research Communications, 496(4): 1263-1268. https://doi.org/10.1016/j.bbrc.2018.01.183

Franco N.M.B. (2007) Cálculo Numérico. São Paulo: Pearson Education. 520 p.

Hirsch C. (2007) Numerical Computation of Internal and External Flows. Great Britain: Elsevier. $715 \mathrm{p}$.

Junior S.C.F. (2003) Modelos de Reação Difusão para o Crescimento de Tumores. Tese de Doutorado, Programa de Pós-Graduação em Física. Universidade Federal de Minas Gerais, Instituto de Ciências Exatas, Departamento de Física, Belo Horizonte, Minas Gerais.

Kansal A.R., Torquato S., Harsh IV G.R., Chiocca E.A. \& Deisboeck T.S. (2000) Cellular automaton of idealized brain tumor growth dynamics. Bio Systems, 55: 119-127. https://doi.org/10.1016/S0303-2647(99)00089-1

LeSar R. (2013) Introduction to Computational Materials Science: Fundamentals to Applications. New York: Cambridge University Press. 427 p.

Murray J.D. (2003) Mathematical Biology II: Spatial Models and Biomedical Applications. $3^{\circ}$ edition. Heidelberg: Springer. 814 p.

Nelson B.L. (2010) Stochastic Modeling: Analysis and Simulation. New York: Dover. 338 p.

Ribeiro T.C.S. (2011) Simulação Computacional da Microestutura das Transformções por Nucleação e Crescimento. Tese de Doutorado, Programa de Pós-Graduação em Engenharia Metalúrgica. Universidade Federal Fluminense, Centro Tecnológico, Escola de Engenharia Industrial Metalúrgica de Volta Redonda, Volta Redonda, Rio de Janeiro. 
Rockne R., Alvord E.C., Rockhill J.K. \& Swanson K.R. (2009) A mathematical model for brain tumor response to radiation therapy. Journal of Mathematical Biology, 58(4-5): 561-578. https://doi.org/10.1007/s00285-008-0219-6

Rodríguez C.R., Calvo G.F., Ramis-Conde I. \& Belmonte-Beitia J. (2017) Stochastic modelling of slow-progressing tumors: Analysis and applications to the cell interplay and control of lowgrade gliomas. Communications in Nonlinear Science and Numerical Simulation, 49: 6380. https://doi.org/10.1016/j.cnsns.2017.02.008

Smolle J.S.H. (1993) Computer simulation of tumor cell invasion by a stochastic. Journal of Theoretical Biology, 160: 63-72. https://doi.org/10.1006/jtbi.1993.1004

Swanson K.R., Alvord J. \& Murray J.D. (2000) A quantitative model for differential motility of gliomas in grey and white matter. Cell Proliferation, 33(5): 317-329. https://doi.org/10.1046/j.1365-2184.2000.00177.x.

Swanson K.R., Bridge C., Murray J.D. \& Alvord E.C. (2003) Virtual and real brain tumors: Using mathematical modeling to quantify glioma growth and invasion. Journal of the Neurological Sciences, 216(1): 1-10. https://doi.org/10.1016/j.jns.2003.06.001

Tanaka M.L., Debinski W. \& Puri I.K. (2009) Hybrid mathematical model of glioma progression. Cell Proliferation, 42(5): 637-646. https://doi.org/10.1111/j.1365-2184.2009.00631.x

Tracqui P., Woodward D.E., Cook J., Cruywagen G.C., Murray J.D. \& Alvord E.C. (1995) A mathematical model of glioma growth: the effect of chemotherapy on spatio-temporal growth. Cell proliferation, 28: 17-31. https://doi.org/10.1111/j.1365-2184.1995.tb00036.x

Unkelbach J., Menze B.H., Konukoglu E., Dittmann F., Le M., Ayache N. \& Shih H.A. (2014) Radiotherapy planning for glioblastoma based on a tumor growth model: Improving target volume delineation. Physics in Medicine and Biology, 59(3): 747-770. https://doi.org/10.1088/0031-9155/59/3/747

Ventura H., Alves A.L.M., Assis W.L.S. \& Rios P.R. (2018) Influence of an exclusion radius around each nucleus on the microstructure and transformation kinetics. Materialia, 2: 167-175. https://doi.org/10.1016/j.mtla.2018.07.009

Yoriyaz H. (2009) Método de Monte Carlo: princípios e aplicações em Física Médica. Revista Brasileira de Física Médica, 3(1): 141-149. http://dx.doi.org/10.29384/rbfm.2009.v3.n1.p141-149 